\title{
Excess noise factor in large area avalanche photodiodes for different temperatures
}

\author{
L.M.P. Fernandes ${ }^{\mathrm{a}}$, J.A.M. Lopes ${ }^{\mathrm{a}, \mathrm{b}, *}$, J.M.F. dos Santos ${ }^{\mathrm{a}}$ \\ ${ }^{a}$ Departamento de Física, Universidade de Coimbra, P-3004-516 Coimbra, Portugal \\ ${ }^{\mathrm{b}}$ Instituto Superior de Engenharia de Coimbra, Rua Pedro Nunes, Quinta da Nora, Apartado 4065, P-3030-199 Coimbra, Portugal
}

Received 20 January 2004; received in revised form 3 May 2004; accepted 10 May 2004

Available online 15 June 2004

\begin{abstract}
The excess noise factor (ENF) of a large area avalanche photodiode was measured as a function of gain for different temperatures, in the -40 to $27^{\circ} \mathrm{C}$ range. Results show that ENF does not depend significantly with temperature attaining values of about 1.8 and 2.3 for gains of 50 and 300 , respectively.
\end{abstract}

(C) 2004 Elsevier B.V. All rights reserved.

PACS: $29.40 . \mathrm{Wk} ; 85.60 . \mathrm{Dw} ; 85.60 . \mathrm{Jb}$

Keywords: Large area avalanche photodiodes; Excess noise factor; Energy resolution

Large-area avalanche photodiodes (LAAPD) are compact, simple to operate, monolithic devices made of silicon $\mathrm{p}-\mathrm{n}$ junctions. When a reverse high voltage is applied to the LAAPD, the internal electric field increases with depth presenting a maximum around the $\mathrm{p}-\mathrm{n}$ junction and reaching values high enough to allow electron multiplication by impact ionization [1,2]. An incident photon produces electron-hole pairs and the resulting electrons are accelerated towards the $\mathrm{n}^{+}$contact, undergoing avalanche multiplication due to the high electric field around the junction. Typical gains of several hundred can be achieved in this process.

*Corresponding author. Tel.: + 351-239-790300; fax: + 351239-790201.

E-mail address: zematias@isec.pt (J.A.M. Lopes).
The application of LAAPDs to direct X-ray detection has been investigated [1-4], mainly to measure charge carrier properties of the device, using X-rays as a reference for visible light measurements $[2,3,5,6]$.

The LAAPD dark current affects the achieved detector energy resolution and limits the minimum detectable energy. As dark current is strongly reduced with decreasing temperature, the operation of LAAPDs at reduced temperatures results in improved performance [7-9].

The performance of a standard LAAPD for Xray and visible-light detection was investigated as a function of the temperature. Dark current, the achieved gain, energy resolution, and minimum detectable energy in X-ray detection were measured for different temperatures [8,11]. Measuring at the same time both $\mathrm{X}$-ray and visible-light pulse 
signals, the excess noise factor (ENF) can be determined [3].

The energy resolution obtained with LAAPDs is mainly limited by three factors: the statistical contribution associated with the number of primary electron-hole pairs and the avalanche process; gain non-uniformity across the diode detection area; noise of the diode-preamplifier system. The variance associated to the statistical factor can be expressed in terms of the number of primary electrons as:

$\sigma_{\text {st }}^{2}=\sigma_{n}^{2}+N \sigma_{A}^{2} / M^{2}$

where $N$ is the number of primary electrons and $\sigma_{n}^{2}$ the correspondent variance, $M$ is the LAAPD gain and $\sigma_{A}^{2}$ the variance of the gain. In this equation, the first term describes the statistical fluctuation of the primary electrons and the second one the fluctuations of the avalanche gain in the LAAPD. Defining the excess noise factor as

$F=1+\sigma_{A}^{2} / M^{2}$

Eq. (1) can be rewritten as

$\sigma_{\mathrm{st}}^{2}=\sigma_{n}^{2}+N(F-1)$.

In particular, for light pulse detection, the variance of primary electrons is described by Poisson statistics

$\sigma_{n}^{2}=N$.

This way, the statistical limitation of the energy resolution is given by

$\sigma_{\mathrm{st}}^{2}=N F$.

Since all the diode area is illuminated, local gains are averaged out and the non-uniformity contribution to the peak broadening can be excluded. Assuming a Gaussian shape of the detected peak, the energy resolution $\Delta E$ of the peak due to the light pulse can be described by the following equation, expressed in $\mathrm{keV}$ :

$\Delta E^{2}=(2.355)^{2} F E \varepsilon+\Delta_{n}^{2}$.

In Eq. (6), $F$ is the ENF, $E$ the energy of the light peak in $\mathrm{keV}, \varepsilon$ the energy per electron-hole pair creation ( $3.6 \mathrm{eV}$ for silicon) and $\Delta_{n}$ the noise contribution (FWHM) of the diode-preamplifier system, in $\mathrm{keV}$. Since X-rays are used as an energy reference for the light signal, $E$ is normalized to the energy of the X-ray signal. $\Delta_{n}$ is determined with a pulse generator. The ENF is then obtained measuring at the same time the signals due to Xrays (for example, $5.4 \mathrm{keV}$ from a ${ }^{54} \mathrm{Mn}$ source), the light pulse and the test pulse. The present method was previously used for the ENF determination at room and LN2 temperatures $[3,12]$. Fig. 1 presents a typical obtained spectrum, showing all necessary parameters.

The used LAAPD is a "Deep UV 500 Windowless series" from Advanced Photonix Inc. [10] with a 16-mm-diameter active area. It was operated in a light-tight box to shield it from ambient light. The box also provides thermal insulation and temperature is controlled through continuous flow of cooled gaseous nitrogen. Temperature stabilization was achieved within $\pm 0.5^{\circ} \mathrm{C}$.

The X-ray signal was obtained from a ${ }^{54} \mathrm{Mn}$ source giving $5.4 \mathrm{keV} \mathrm{X}$-rays. The light signal was provided by a light emitting diode (LED) with peak emission at $635 \mathrm{~nm}$. The LED was connected to a $50 \Omega$ resistance and supplied by a "LEDpulser" producing pulses of 6-500 ns width and up to $10 \mathrm{~V}$ amplitude. A light guide was used to carry the light pulses to the LAAPD.

The LAAPD signals were fed through a lownoise charge preamplifier (Canberra 2004, with a sensitivity of $45 \mathrm{mV} / \mathrm{MeV}$ ) to a linear amplifier (HP 5582A, with gain of $16-128$ and $200 \mathrm{~ns}$ integration and differentiation time constants) and were pulse-height analyzed by a 1024-channel multi-channel analyzer (Nucleus PCA II).

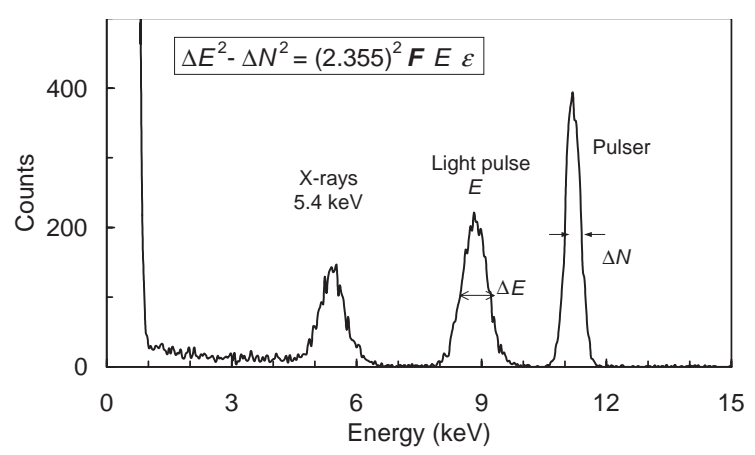

Fig. 1. Typical energy spectrum for the ENF determination. Three different peaks are shown: the X-ray peak produced by $5.4 \mathrm{keV}$ X-rays from a ${ }^{54} \mathrm{Mn}$ radioactive source, the light peak produced by a LED pulse and the peak from a pulse generator. 


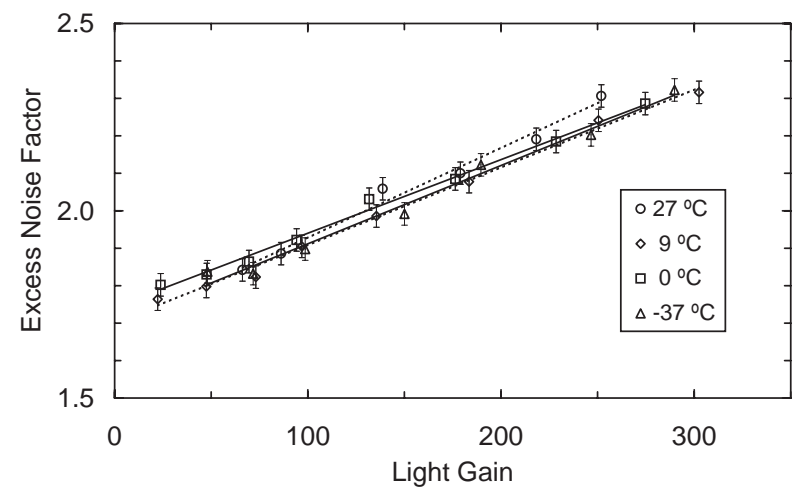

Fig. 2. Excess noise factor as function of gain for different temperatures.

To measure all quantities at the same time, for different temperatures, the LAAPD was simultaneously irradiated with X-rays and visible-light. The LED intensity was chosen to produce a pulse with an amplitude equivalent to about $9 \mathrm{keV} \mathrm{X-}$ rays. The test pulser was fed through the preamplifier test input. Fig. 2 presents the ENF, calculated according to Fig. 1, as function of the LAAPD gain for different temperatures.

As shown in Fig. 2, ENF increases almost linearly with gain and does not have significant variations with temperature. The present results are in good agreement with previous measurements, performed at room temperature [3] and LN2 temperature [12].

In previous works, energy resolution dependence on temperature was observed and could be related to variation of both ENF and dark current-derived noise level. It is now clear that this dependence is not related with the ENF. Thus, decreasing temperature will significantly improve the signal-to-noise ratio, leading to lower-energy resolution.

\section{Acknowledgements}

This work was carried out under Project POCTI/FNU/41720/01 of the Atomic and Nuclear
Instrumentation Group of the Physics Department, University of Coimbra.

\section{References}

[1] E.M. Gullikson, E. Gramsch, M. Szawlowski, Appl. Opt. 34 (1995) 4662.

[2] J.P. Pansart, Nucl. Instr. and Meth. A 387 (1997) 186.

[3] M. Moszynski, M. Szawlowski, M. Kapusta, M. Balcerzyk, D. Wolski, IEEE Trans. Nucl. Sci.-NS 47 (2000) 1297.

[4] L.M.P. Fernandes, J.A.M. Lopes, J.M.F. dos Santos, C.A.N. Conde, X-ray Spectrom. 30 (2001) 164.

[5] M. Moszynski, M. Kapusta, M. Szawlowski, D. Wolski, I. Wegrzecka, M. Wegrzecki, IEEE Trans. Nucl. Sci.-NS 48 (2001) 1205.

[6] C.P. Allier, H. Valk, V.R. Bom, R.W. Hollander, C.W.E. van Eijk, IEEE Trans. Nucl. Sci.-NS 45 (1998) 576.

[7] V. Solovov, et al., IEEE Trans. Nucl. Sci.-NS 47 (2000) 1307.

[8] L.M.P. Fernandes, J.A.M. Lopes, J.M.F. dos Santos, C.A.N. Conde, Nucl. Instr. and Meth. B 213 (2004) 267.

[9] J.A.M. Lopes, L.M.P. Fernandes, J.M.F. dos Santos, R.E. Morgado, C.A.N. Conde, Nucl. Instr. and Meth. A 504 (2003) 331.

[10] Advanced Photonix Inc., 1240Av.Acaso, Camarillo, CA 93012, USA.

[11] L.M.P. Fernandes, J.A.M. Lopes, J.M.F. dos Santos, et al., LAAPD low temperature performance in X-ray and visible-light detection, IEEE Trans. Nucl. Sci., to be published in August 2004, (Vol. 51, issue 4).

[12] M. Moszynski, M. Czarnacki, M. Szawlowski, B.L. Zhou, M. Kapusta, D. Wolski, P. Schotanous, IEEE Trans. Nucl. Sci.-NS 49 (2002) 971. 